\title{
Effect of Feeding Probiotics on Rats' Immunity and Health Conditions during Pregnancy
}

\author{
Abdul-Rahman A. Ali ${ }^{1}$, Ahmed M. M. Metwally ${ }^{1 *}$, Amal H. Mahmoud ${ }^{2}$ Hadil F. Attia $^{2}$ \\ ${ }^{1}$ Dairy Science and Technology Department, Faculty of Agriculture, Cairo University, Giza, Egypt; ${ }^{2}$ Food Technology Research \\ Institute, Agricultural Research Center, Giza, Egypt. \\ E-mail: ahmmetwally@hotmail.com
}

Received August $16^{\text {th }}, 2010$; revised November $25^{\text {th }}, 2010$; accepted December $24^{\text {th }}, 2010$.

\begin{abstract}
Pregnancy is associated with down regulating cell-mediated immunity (T-Lymphocyte) which would lead for increasing susceptibility to viral and bacterial infection, therefore it is expected that feeding probiotic bacteria would help in strengthening pregnant immunity. To test the effect of probiotics feeding on immunity during pregnancy, rats were fed on four different diets, a basal diet (the control) or basal diet fortified with different probiotics which were yogurt (Streptococcus thermophilus EMCC 1043 and Lactobacillus delbruckii subsp bulgaricus EMCC 1102) (G1), yogurt plus Bifidobacterium breve (G2), and yogurt plus Bifidobacterium breve plus Lactobacillus paracasei (G3). Feeding started before mating, during gestation and after parturition. Probiotics particularly (G2) and (G3) improved significantly rats' body weight gain over the control during gestation and after parturition and their off-spring. G2 and G3 significantly lowered total serum cholesterol than the control. Low Density Lipoprotein (LDL)-fraction decreased insignificantly during gestation but the difference was significant after parturition. Probiotic feeding enhanced leukocyte production which was differentiated more into lymphocytes than phagocytes particularly on third week of gestation and after parturition. $\mathrm{CD}_{4}{ }^{+}$counts in the control rats suffered a decrease on pregnancy and the decrease continued during gestation period and after parturition. On the other hand, probiotic feeding helped $\mathrm{CD}_{4}$ to overcome the decrease occurred on pregnancy to give counts on the third week and after parturition higher than before pregnancy. $\mathrm{CD}_{8}{ }^{+}$counts were also increased with probiotics feeding as compared to the control which suffered continuous decrease during pregnancy. Therefore, probiotics feeding improved pregnant rats' general health, lipid profile and both sides of immunity.
\end{abstract}

Keywords: Immunity, Lipoproteins, Probiotics, Pregnancy

\section{Introduction}

During pregnancy, a state of selective immune tolerance is achieved which causes maternal circulating immune cells undergo modifications in their counts, phenotypes, functions and ability to produce soluble factors (e.g. cytokines) to establish the ultimate goal of a successful pregnancy. In mammalian, the immune system fulfill this needs by down regulating potentially dangerous T-cells mediated immune responses and activating certain components of the innate immune system such as monocyte and neutrophil. Changes occurring in both sides of immunity on pregnancy have been studied and the results were contradicting either in total number or percent distribution of subsets blood leucocytes. Discrepancies in the counts of $\mathrm{CD}_{8}{ }^{+}-\mathrm{T}$ and $\mathrm{CD}_{4}{ }^{+}$-T cells during pregnancy were reported $[1,2]$. Most important reported changes were the increase in the percent and count of granulocyte and monocyte and complement proteins, this was to set a balance between their increase and the reduction of T-cells and NK and Type 1 cytokines [3]. It was postulated that to insure normal pregnancy, percentage of lymphocyte cells producing Ifn- $\gamma$ should not exceed a certain maximum depending on the species. The percent of lymphocyte producing Ifn- $\gamma$ and IL-4 cells are slightly increased in rats to give immune response of (Th1). But in humans, Ifn- $\gamma$ producing cells decreased to give (Th2) cytokine humoral response, releasing IL-4, IL-5 and IL-10 to regulate antibody productions to strengthen humoral immunity [4]. The mechanisms on pregnancy by which the immune system modulates $\mathrm{T}$-cell reactivity and production of cytokines were suggested to depend on several factors such as, production of estrogen and progesterone hormones, the presence of an immune suppressive factor (specific glycoprotein) and more important 
the existence of effectors-T-cell with regulatory functions (Tregs) carrying $\mathrm{CD}_{25}{ }^{+}-\mathrm{T}$. These are potent suppressors of T-cells responses and can protect tissues from cell-mediated destruction. Tregs concentration progressively increased at each stage of human pregnancy and they appeared in rats after two days after mating [5]. Though, there was no scientific evidence that pregnant women are more susceptible to infections, the down regulation of T-lymphocyte is considered the basis for increasing susceptibility to viral infection and intercellular pathogen e.g. listeria. Therefore, any practice that would enhance production of both sides of immunity, the innate as well as cell- mediated without any detrimental effect on the fetus would be welcomed.

Beside the change in immunity, there was an increase in serum cholesterol on pregnancy which was thought to be correlated to the increase in the risk of cardiovascular disease [6].

Consuming probiotics, microorganisms that confer health benefits, is an old practice that humans accustomed to. Many health benefits have been reported, such as immunomodulating effect which helps persons' immunity in retarding number of diseases [6-8], reduction of serum cholesterol $[9,10]$, enhancing body absorption of minerals and many other health benefits. However, types of health benefits as well as their mechanisms are microorganism (type \& strain) and dose dependent. As for the mechanism, not all lactic acid bacteria (LAB) which are the major probiotics stimulate the immune system in the same way [11]. For example, B. bifidum increased total secretion of major immunoglobulin (Ig) isotypes and the number of IgA secreting cells [12]. $L b$. helveticus activity in casein permeate resulted in interaction with monocyte-macrophage and T-helper cells especially Th1-like cells $[11,13,14]$. Lb. casei, Lb. acidophilus and yoghurt enhanced the number of $\operatorname{IgA}$ producing cells and mucosal immune response [7-9]. Lb. acidophilus and $B$. bifidum enhanced phagocyte activity of granulocyte and monocyte [8]. Bifidobacterium thermophilus enhanced cytotoxic activity of NK cells in spleen and mesenteric lymph node in normal and tumor bearing mice. $L b$. casei inhibited IgE production; enhanced Th1 cell associated cytokine production and suppressed $\mathrm{Th}_{2-}$ cytokine production [16]. Lb. plantarum stimulated activity of B cells population of splenocyte. $L b$. casei and Lb. acidophilus increased IgM forming cell in spleen [7, 8]. B. breve strain yakult and $L b$. casei resulted in the protection of mice from lethal STEC infections by inhibiting the production of Shiga toxin (Stx) in the intestine $[18,19]$.

Therefore, this work was carried out to study the effect of probiotics on the immunity, health conditions and lipid profile of rats on pregnancy. The effect of probiotics feeding on leukocyte count, lymphocyte percent, phagocyte percent and lymphocyte $\mathrm{CD}_{4}^{+}-\mathrm{T}$ and $\mathrm{CD}_{8}{ }^{+}-\mathrm{T}$ cells, serum lipid profile and body weight during gestation and postpartum were studied.

\section{Materials and Methods}

\subsection{Bacterial Strains}

Streptococcus thermophilus EMCC 1043 and Lactobacillus delbruckii subsp bulgaricus EMCC 1102, Bifidobacterium breve and Lactobacillus paracasei obtained from Cairo Mircen (Ain Shams University, Cairo, Egypt) and were used for milk fermentation.

\subsection{Manufacturing of Yoghurt}

Buffalos' Milk from Faculty of Agriculture, Cairo University, Egypt was heated at $90^{\circ} \mathrm{C} / 15 \mathrm{~min}$, cooled to $40^{\circ} \mathrm{C}$, inoculated with $1.5 \%$ of the culture and was left for coagulation in a $40^{\circ} \mathrm{C}$ incubator. Three batches of fermented milk were processed using the regular yoghurt starter plus different probiotic cultures distributed as follows:

- Group 1: 1.5\% Sc. thermophilus + 1.5\% Lb. delbruckii subsp bulgaricus.

- Group 2: 1.5\% Sc. thermophilus + 1.5\% Lb. delbruckii subsp bulgaricus $+2 \%$ B. breve.

- Group3: 1.5\% Sc. thermophilus + 1.5\% Lb. delbruckii subsp bulgaricus $+1 \%$ B. breve $+1 \% L b$. paracasei.

Table 1 shows the chemical composition of the prepared yoghurt.

\subsection{The Experimental Diets}

Three experimental diets were prepared by mixing 97\% of the basal diet with $3 \%$ of each of the above probiotic yogurt; these three groups were compared with a negative control group fed on the basal diet composed of $20 \%$ casein, 10\% corn oil, 5\% cellulose, 1\% vitamin mix, 4\% minerals and $60 \%$ starch. Table 2 illustrates the composition of the used diets.

\subsection{Microbiological Analysis of Prepared Yogurt}

Lb. delbruckii subsp bulgaricus, Sc. thermophilus and $L b$. paracasei were counted on MRS agar after incubation at $40-45^{\circ} \mathrm{C} / 48 \mathrm{hr}$. Bifidobacteria was counted on modified MRS agar to which the following fortifications [20] were added at a rate of $5 \mathrm{ml}$ for every $100 \mathrm{ml}$ of modified MRS agar: Neomycine sulphate $(0.80 \%$, w/v), Paromomycine sulphate $(0.20 \%$, w/v), Nalidixic acid. $(0.30 \%$, $\mathrm{w} / \mathrm{v})$ and Lithium chloride $(6.00 \%, \mathrm{w} / \mathrm{v})$. Plates were incubated under anaerobic condition at $37^{\circ} \mathrm{C}$ for $72 \mathrm{hr}$. The bacterial counts of the three experimental yogurts are in Table 3. 
Table 1. Chemical composition of yoghurt.

\begin{tabular}{cccccc}
\hline Treatment & $\begin{array}{c}\text { Protein, } \\
\%\end{array}$ & $\begin{array}{c}\text { Fat, } \\
\%\end{array}$ & $\begin{array}{c}\text { PH, } \\
\%\end{array}$ & $\begin{array}{c}\text { Acidity, } \\
\%\end{array}$ & $\begin{array}{c}\text { Total Solids, } \\
\%\end{array}$ \\
\hline Regular yogurt & 5 & 7.5 & 4.91 & 0.8 & 18.50 \\
Yogurt + Lb. Paracasei & 4.8 & 7.3 & 4.47 & 0.85 & 18.45 \\
$\begin{array}{c}\text { Yogurt + B. breve } \\
\text { Yogurt + Lb. paracasei }+ \\
\text { B. breve }\end{array}$ & 4.8 & 7.5 & 4.56 & 0.9 & 18.51 \\
\hline
\end{tabular}

Table 2. Composition of diets used in the treatments.

\begin{tabular}{cc}
\hline Groups & Experimental diets (per day) \\
\hline $\begin{array}{c}\text { Negative } \\
\text { control }\end{array}$ & $30 \mathrm{~g}$ basal diet (for each rat) \\
Group 1 & $\begin{array}{c}26.55 \mathrm{~g} \text { basal diet }+3.45 \mathrm{~g} \text { yogurt with } 1.5 \% \text { Sc. thermo- } \\
\text { philus }+1.5 \% \text { Lb. delbrueckii subsp bulgaricus. }\end{array}$ \\
& $\begin{array}{c}26.55 \mathrm{~g} \text { basal diet }+3.45 \mathrm{~g} \text { yogurt with } 1.5 \% \text { Sc. thermo- } \\
\text { philus }+1.5 \% \text { Lb. delbrueckii subsp bulgaricus }+2 \% \text { B. } \\
\text { breve. }\end{array}$ \\
Group 3 & $\begin{array}{l}26.55 \mathrm{~g} \text { basal diet }+3.45 \mathrm{~g} \text { yogurt with } 1.5 \% \text { Sc. thermophi- } \\
\text { lus }+1.5 \% \text { Lb. delbrueckii subsp bulgaricus }+1 \% \text { B. breve } \\
\end{array}$ \\
\hline
\end{tabular}

\subsection{Animal Feeding Experiments}

Thirty Two Albino rats (24 Females and 8 Males) weighting $200 \mathrm{~g}-260 \mathrm{~g}$ (average weights of each group are shown in Table 4) obtained from Animal House of Food Technology and Research Institute, Agriculture Research Center, Giza, Egypt. They were kept under normal healthy conditions $\left(25^{\circ} \mathrm{C}-27^{\circ} \mathrm{C}\right.$ and $75 \%$ humidity) and fed on basal diet for three days adaptation before mating. After adaptation, rats were randomly divided into four groups; each group contained 2 male and 6 female rats and was left for mating, and assigned to the experimental diet. After twelve days of mating, male rats were isolated.

\subsection{Body Weight}

Body weight of individual rats was recorded every week.

\subsection{Blood Sampling}

Blood samples were collected from the eye vein of the rat under diethyl ether anesthesia and composite samples

Table 3. Bacterial counts of the experimental yogurts

\begin{tabular}{cccc}
\hline \multirow{2}{*}{ Microorganisms } & \multicolumn{3}{c}{ Microbial count $\mathbf{1 0}^{\mathbf{6}} \mathbf{c f u} / \mathbf{g}$} \\
\cline { 2 - 4 } & Group 1 & Group 2 & Group 3 \\
\hline $\begin{array}{c}\text { Lb. delbruckii subsp } \\
\text { bulgaricus }\end{array}$ & 1.3 & 1.1 & 1.1 \\
Sc. thermophilus & 1.8 & 1.2 & 1.1 \\
B. breve & - & 1.4 & 1.3 \\
Lb. paracasei & - & - & 1.5 \\
\hline
\end{tabular}

Table 4. The effect of feeding probiotics on rats body weight (g) during pregnancy and after parturition and their offspring.

\begin{tabular}{ccccc}
\hline Treatments & $\begin{array}{c}\text { Negative } \\
\text { control }\end{array}$ & Group 1 & Group 2 & Group 3 \\
\hline \multirow{5}{c}{ Rats } & & & \\
Before pregnancy & 223.00 & 261.00 & 234.33 & 242.33 \\
& $\pm 1.53 \mathrm{~d}$ & $\pm 2.08 \mathrm{c}$ & $\pm 2.96 \mathrm{c}$ & $\pm 1.45 \mathrm{~d}$ \\
& 310.00 & 353.33 & 390.67 & 401.6 \\
$\mathbf{1}^{\text {st }}$ week pregnancy & $\pm 5.77 \mathrm{~b}$ & $\pm 13.33 \mathrm{~b}$ & $\pm 7.22 \mathrm{a}$ & $\pm 7.84 \mathrm{~b}$ \\
& 325.00 & 372.33 & 403.33 & 418.33 \\
$\mathbf{2}^{\text {nd }}$ week pregnancy & $\pm 8.74 \mathrm{ab}$ & $\pm 9.84 \mathrm{ab}$ & $\pm 7.26 \mathrm{a}$ & $\pm 4.41 \mathrm{ab}$ \\
& 342.33 & 397.00 & 410.33 & 433.67 \\
$\mathbf{3}^{\text {rd }}$ week pregnancy & $\pm 10.40 \mathrm{a}$ & $\pm 9.02 \mathrm{a}$ & $\pm 5.49 \mathrm{a}$ & $\pm 8.76 \mathrm{a}$ \\
& 285.00 & 297.33 & 327.00 & 370.00 \\
After parturition & $\pm 8.66 \mathrm{c}$ & $\pm 18.41 \mathrm{c}$ & $\pm 12.74 \mathrm{~b}$ & $\pm 5.77 \mathrm{c}$ \\
& 24.208 & 37.266 & 24.655 & 19.583 \\
LSD at 5\% & Mice & & \\
& 23.00 & 27.67 & 16.67 & 26.67 \\
On birth & $\pm 1.527 \mathrm{~b}$ & $\pm 0.882 \mathrm{~b}$ & $\pm 0.882 \mathrm{~b}$ & $\pm 1.453 \mathrm{~b}$ \\
& 31.33 & 33.33 & 24.33 & 36.00 \\
1st week & $\pm 0.882 \mathrm{a}$ & $\pm 0.882 \mathrm{a}$ & $\pm 1.202 \mathrm{a}$ & $\pm 0.577 \mathrm{a}$ \\
LSD at 5\% & 4.897 & 3.463 & 4.139 & 4.341 \\
\hline
\end{tabular}

Control group: Fed on basal diet. Group 1: Fed on yogurt culture. Group 2: Fed on yogurt culture $+B$. breve. Group 3: Fed on yogurt culture $+L b$. paracasei \& B .breve.

were prepared for analysis. A sample was taken into tubes containing 6\% EDTA as anti-coagulant for lymphocytes subsets analysis, and another sample was taken and centrifuged at $3500 \mathrm{rpm} / 10 \mathrm{~min}$ to obtain the serum which was kept frozen until analysis. Blood samples were taken, before pregnancy and on first, second and third week of pregnancy and after parturition.

\subsection{Blood Analysis}

Plasma total cholesterol, high density lipoprotein (HDL) and triglycerides were determined using EnzymaticColorimetric. Tender kits (ELI TECH-France). Low density Lipoprotein (LDL) and Very Low Density Lipoprotein (VLDL) cholesterol were estimated by the following equations:

$$
\begin{aligned}
& \mathrm{LDL}=\text { total cholesterol }- \text { triglyceride } / 5-\mathrm{HDL} \\
& \mathrm{VLDL}=\text { triglyceride } / 5
\end{aligned}
$$

\subsection{Immunological Analysis}

White Blood cells were counted by Haemocytometer slide. Flow cytometric analysis of leukocyte subsets was performed using COULTER EPICSR XL (Germany).

\subsection{Statistical Analysis}

Analysis were in triplicates and statistical analysis was carried out according to Fisher, (1970) [21]. Least significant difference (LSD) test was used to compare the significant differences between means of treatment [22]. The statistical package for social science (S.P.S.S., 2003) 
program version 12 was used for all analysis [23].

\section{Results and Discussion}

Twenty four female rats divided into four groups were fed on experimental diets before mating and through pregnancy till parturition. Table 4 presents rats' body weight during pregnancy and after parturition. During gestation, body weight progressively increased and on the third week of gestation, there was significant increase in body weight of all groups.

The experimental groups showed higher weight gain than the control. The weight gain was 191, 176, 136 and 119 grams in group three, two, one and control, respectively. After parturition, the weight decreased but rats did not return to their original weight before pregnancy. The body weight gain after parturition was higher in groups three and two (128 and 93 grams) than group one and the control (36 and 62 grams), respectively. Table 4 shows rats off spring body weight gain during two weeks of lactation. The weight increased significantly, but the weight gain was higher in mice fed on diet of group three $(9.3 \mathrm{~g})$ followed by group two and the control both gained $8 \mathrm{~g}$ while group one gained only $6 \mathrm{~g}$. Therefore, feeding rats on $B$. breve, $L b$. paracasei plus yogurt culture or on $B$. breve plus yogurt culture enhanced rats body weight gain during gestation and after parturition over the basal control diet.

Table 5 reports rats' serum lipid changes throughout the experiment. Though literature pointed out that total and LDL cholesterol and triglycerides levels progressively increase during gestation [6], feeding probiotics showed different trend. Concentration of rat's total cholesterol increased on pregnancy and this continued to reach a maximum concentration either on the first week in groups one and three (from 66 and 65 into 71 and 80 $\mathrm{mg} / \mathrm{dl}$, respectively) or on the second week as in the control and group two (from 69.3 and 56 into 90 and 62 $\mathrm{mg} / \mathrm{dl}$, respectively) then started to decline till parturition. The decline rate was slight in control rats and group one but was more pronounced in the culture fed rats (group two and three). On the third week of gestation, groups fed on probiotics contained significantly lower cholesterol concentration than before pregnancy (56 and 56.3 $\mathrm{mg} / \mathrm{dl}$ compared to 66 and 65 in groups 1 and 3, respectively). Not only that, but also, the reduction of cholesterol concentration continued to be significant after parturition (47.6, 46.3 and 44.6 compared to 66,56 , and 65 before pregnancy in groups 1,2 and 3, respectively). Cholesterol concentration in the control rats followed the literature trend where the concentration increased on pregnancy and the level during gestation and after parturition continued to be higher than before pregnancy. The concentrations were $69.3,84$ and $77.6 \mathrm{mg} / \mathrm{dl}$ before pregnancy, on third week of gestation and after parturition, respectively.

Low-density lipoprotein (LDL) concentration trend was similar to total cholesterol. LDL in the probiotic groups increased on pregnancy then started to decline on the third week of gestation to be insignificantly lower than the level before pregnancy. The LDL concentrations were $32.3,40.6,33.3$ and $44.6 \mathrm{mg} / \mathrm{dl}$ before pregnancy and these were changed on third week of gestation into $30.3,39.3,28$ and $41.3 \mathrm{mg} / \mathrm{dl}$ in the control and groups 1 , 2 and 3, respectively. After parturition, LDL level was lower than before pregnancy in all groups, the difference was significant in the control and groups two and three.

The beneficial effect of probiotic consumption was also manifested in VLDL concentration which increased on first week of gestation, but the increase was insignificant, followed by a decline to give concentrations lower than before pregnancy level. Concentrations of $12.3,10.3$ and $15.3 \mathrm{mg} / \mathrm{dl}$ before pregnancy declined to $8.3,7.6$ and $10 \mathrm{mg} / \mathrm{dl}$ on third week of gestation in groups 1,2 and 3, respectively. On the contrary, VLDL of the control rats increased significantly on pregnancy (from 15 into $19.3 \mathrm{mg} / \mathrm{dl}$ ) and this trend continued during gestation period. After parturition the level was still higher than before pregnancy. High density lipoprotein (HDL) was kept almost unchanged in probiotic groups.

Rats' serum Triglycerides (TG) showed a trend similar to that of total cholesterol, the concentration significantly increased on pregnancy and the increase continued to the second week of gestation. The level started to decline on the third week of gestation, the rate of decline was slight in the control and was significant in the probiotic groups. After parturition, the TG concentration was insignificantly higher in the control rats but was significantly lower in probiotic groups than before pregnancy.

Results pointed out that Probiotics feeding improved serum total cholesterol, LDL, VLDL and triglycerides, thus overcoming their rise properties encountered on pregnancy.

Table 6 presents rats leucocytes count development during pregnancy and after parturition as affected by probiotics feeding. In general, the count increased on pregnancy till parturition but the trend of the increase through gestation varied according to the group. The control showed an insignificant decrease in the count on first week of pregnancy (from 6186 into 5556 cells $/ \mathrm{ml}$ ) followed by a significant increase to reach 8200 cells on third week of gestation. After parturition, the counts were lower than before pregnancy, they were 6186 before pregnancy and became 5401 after parturition. On the other hand, leukocyte counts were significantly increased in group two (from 7150 into 9483) and three (from 9300 into 9850) and insignificantly in group one on pregnancy. 
Table 5. Effect of feeding probiotics on rats' lipid profile (mg/dl) during pregnancy and after parturition.

\begin{tabular}{|c|c|c|c|c|c|c|c|c|c|c|c|}
\hline \multirow[b]{2}{*}{ Treatments } & \multicolumn{6}{|c|}{ Negative control } & \multicolumn{5}{|c|}{ Group 1} \\
\hline & CHO & TG & \multicolumn{2}{|c|}{ LDL } & HDL & VLDL & CHO & TG & LDL & HDL & VLDL \\
\hline Before pregnancy & $\begin{array}{c}69.33 \\
\pm 2.33 \mathrm{c}\end{array}$ & $\begin{array}{c}82.67 \\
\pm 1.45 \mathrm{c}\end{array}$ & \multicolumn{2}{|c|}{$\begin{array}{c}32.33 \\
\pm 1.86 \mathrm{ab}\end{array}$} & $\begin{array}{c}23.33 \\
\pm 1.20 \mathrm{c}\end{array}$ & $\begin{array}{c}15.00 \\
\pm 1.53 \mathrm{c}\end{array}$ & $\begin{array}{c}66.00 \\
\pm 2.08 \mathrm{~d}\end{array}$ & $\begin{array}{c}60.00 \\
\pm 1.73 \mathrm{~b}\end{array}$ & $\begin{array}{c}40.67 \\
\pm 1.45 \mathrm{c}\end{array}$ & $\begin{array}{c}15.67 \\
\pm 0.33 \mathrm{ab}\end{array}$ & $\begin{array}{c}12.33 \\
\pm 0.88 \mathrm{ab}\end{array}$ \\
\hline 1st week pregnancy & $\begin{aligned} & 85.33 \\
\pm & 2.91 \mathrm{ab}\end{aligned}$ & $\begin{array}{r}101.00 \\
\pm 4.58 \mathrm{a}\end{array}$ & \multicolumn{2}{|c|}{$\begin{array}{c}35.33 \\
\pm 1.76 \mathrm{a}\end{array}$} & $\begin{array}{c}33.00 \\
\pm 1.53 \mathrm{~b}\end{array}$ & $\begin{aligned} & 19.33 \\
\pm & 0.88 \mathrm{ab}\end{aligned}$ & $\begin{array}{c}71.00 \\
\pm 2.08 \mathrm{a}\end{array}$ & $\begin{array}{c}74.00 \\
\pm 2.08 \mathrm{a}\end{array}$ & $\begin{aligned} & 41.33 \\
\pm & 0.88 \mathrm{ab}\end{aligned}$ & $\begin{array}{c}13.33 \\
\pm 0.88 \mathrm{bc}\end{array}$ & $\begin{array}{c}13.67 \\
\pm 0.88 \mathrm{a}\end{array}$ \\
\hline 2nd week pregnancy & $\begin{array}{c}90.00 \\
\pm 2.89 \mathrm{a}\end{array}$ & $\begin{array}{l}102.67 \\
\pm 1.45 \mathrm{a}\end{array}$ & \multicolumn{2}{|c|}{$\begin{array}{c}28.67 \\
\pm 1.86 \mathrm{~b}\end{array}$} & $\begin{array}{c}40.67 \\
\pm 1.20 \mathrm{a}\end{array}$ & $\begin{array}{c}21.67 \\
\pm 0.88 \mathrm{a}\end{array}$ & $\begin{array}{c}62.67 \\
\pm 1.45 \mathrm{~b}\end{array}$ & $\begin{array}{c}70.33 \\
\pm 0.88 \mathrm{a}\end{array}$ & $\begin{array}{c}42.00 \\
\pm 1.53 \mathrm{a}\end{array}$ & $\begin{array}{c}17.67 \\
\pm 0.88 \mathrm{a}\end{array}$ & $\begin{aligned} & 13.33 \\
\pm & 0.33 \mathrm{ab}\end{aligned}$ \\
\hline 3rd week pregnancy & $\begin{aligned} & 84.00 \\
\pm & 3.05 \mathrm{ab}\end{aligned}$ & $\begin{array}{c}93.00 \\
\pm 1.15 \mathrm{~b}\end{array}$ & \multicolumn{2}{|c|}{$\begin{array}{c}30.33 \\
\pm 1.45 \mathrm{ab}\end{array}$} & $\begin{array}{c}36.33 \\
\pm 2.40 \mathrm{ab}\end{array}$ & $\begin{array}{c}18.33 \\
\pm 0.33 \mathrm{~b}\end{array}$ & $\begin{array}{c}56.00 \\
\pm 2.08 \mathrm{c}\end{array}$ & $\begin{array}{c}57.67 \\
\pm 1.20 \mathrm{~b}\end{array}$ & $\begin{aligned} & 39.33 \\
\pm & 2.33 \mathrm{abc}\end{aligned}$ & $\begin{array}{c}12.00 \\
\pm 0.58 \mathrm{c}\end{array}$ & $\begin{array}{c}11.00 \\
\pm 0.58 \mathrm{~b}\end{array}$ \\
\hline After parturition & $\begin{array}{c}77.67 \\
\pm 1.76 \mathrm{~b}\end{array}$ & $\begin{aligned} & 86.33 \\
\pm & 2.03 \mathrm{bc}\end{aligned}$ & \multicolumn{2}{|c|}{$\begin{array}{c}20.67 \\
\pm 1.20 \mathrm{c}\end{array}$} & $\begin{array}{c}40.00 \\
\pm 2.08 \mathrm{a}\end{array}$ & $\begin{aligned} & 17.00 \\
\pm & 0.58 \mathrm{bc}\end{aligned}$ & $\begin{array}{c}47.6 \\
\pm 1.45 \mathrm{e}\end{array}$ & $\begin{array}{c}40.33 \\
\pm 1.45 \mathrm{c}\end{array}$ & $\begin{array}{l}36.00 \\
\pm 1.53 \mathrm{bc}\end{array}$ & $\begin{array}{c}16.33 \\
\pm 1.20 \mathrm{a}\end{array}$ & $\begin{array}{c}8.33 \\
\pm 0.88 \mathrm{c}\end{array}$ \\
\hline LSD at $5 \%$ & 8.30 & 7.80 & \multicolumn{2}{|c|}{5.19} & 5.52 & 2.93 & 5.85 & 4.81 & 5.08 & 2.62 & 2.35 \\
\hline \multirow{2}{*}{ Treatments } & \multicolumn{6}{|c|}{ Group 2} & \multicolumn{5}{|c|}{ Group 3} \\
\hline & \multicolumn{2}{|l|}{ CHO } & TG & LDL & HDL & VLDL & CHO & TG & \multicolumn{2}{|c|}{ LDL HDL } & VLDL \\
\hline Before pregnancy & $\begin{aligned} & 56.00 \\
\pm & 0.58 \mathrm{bc}\end{aligned}$ & & $\begin{array}{l}5.33 \\
1.76 \mathrm{~b}\end{array}$ & $\begin{array}{c}33.33 \\
\pm 2.03 \mathrm{~b}\end{array}$ & $\begin{array}{c}14.33 \\
\pm 0.67 \mathrm{a}\end{array}$ & $\begin{array}{l}10.33 \\
\pm 0.88 \mathrm{abc}\end{array}$ & $\begin{array}{c}65.00 \\
\pm 1.73 \mathrm{c}\end{array}$ & $\begin{array}{c}75.00 \\
\pm 1.73 \mathrm{~b}\end{array}$ & $\begin{aligned} & 44.67 \\
\pm & 0.88 \mathrm{bc}\end{aligned}$ & $\begin{array}{c}15.67 \\
\pm 1.20 \mathrm{a}\end{array}$ & $\begin{array}{c}15.33 \\
\pm 0.33 \mathrm{a}\end{array}$ \\
\hline 1st week pregnancy & $\begin{array}{c}58.00 \\
\pm 1.15 \mathrm{ab}\end{array}$ & & $\begin{array}{l}2.67 \\
1.45 \mathrm{a}\end{array}$ & $\begin{array}{c}32.67 \\
\pm 1.45 \mathrm{a}\end{array}$ & $\begin{array}{c}13.00 \\
\pm 1.00 \mathrm{ab}\end{array}$ & $\begin{array}{c}13.33 \\
\pm 0.88 \mathrm{a}\end{array}$ & $\begin{array}{c}80.00 \\
\pm 1.15 \mathrm{a}\end{array}$ & $\begin{array}{c}87.6 \\
\pm 1.45 \mathrm{a}\end{array}$ & $\begin{array}{c}52.67 \\
\pm 1.76 \mathrm{a}\end{array}$ & $\begin{array}{c}9.67 \\
\pm 0.88 \mathrm{~b}\end{array}$ & $\begin{array}{c}16.00 \\
\pm 1.53 \mathrm{a}\end{array}$ \\
\hline 2nd week pregnancy & $\begin{array}{c}62.00 \\
\pm 1.53 \mathrm{a}\end{array}$ & & $\begin{array}{l}7.33 \\
.76 \mathrm{ab}\end{array}$ & $\begin{array}{c}34.33 \\
\pm 0.88 \mathrm{ab}\end{array}$ & $\begin{array}{c}14.00 \\
\pm 1.53 \mathrm{a}\end{array}$ & $\begin{array}{c}12.00 \\
\pm 1.15 \mathrm{ab}\end{array}$ & $\begin{array}{c}73.67 \\
\pm 1.86 \mathrm{~b}\end{array}$ & $\begin{array}{c}77.33 \\
\pm 1.45 \mathrm{~b}\end{array}$ & $\begin{array}{c}47.00 \\
\pm 1.53 \mathrm{~b}\end{array}$ & $\begin{array}{c}15.67 \\
\pm 1.20 \mathrm{a}\end{array}$ & $\begin{array}{c}13.67 \\
\pm 1.86 \mathrm{a}\end{array}$ \\
\hline 3rd week pregnancy & $\begin{array}{c}53.33 \\
\pm 1.45 \mathrm{c}\end{array}$ & & $\begin{array}{l}4.33 \\
2.33 b\end{array}$ & $\begin{array}{c}28.00 \\
\pm 1.53 \mathrm{~b}\end{array}$ & $\begin{array}{c}10.00 \\
\pm 0.58 \mathrm{~b}\end{array}$ & $\begin{aligned} & 8.67 \\
\pm & 1.20 \mathrm{bc}\end{aligned}$ & $\begin{array}{c}56.3 \\
\pm \\
\pm 2.33 \mathrm{~d}\end{array}$ & $\begin{array}{c}67.33 \\
\pm 1.45 \mathrm{c}\end{array}$ & $\begin{array}{c}41.33 \\
\pm 0.88 \mathrm{~cd}\end{array}$ & $\begin{array}{c}13.00 \\
\pm 0.58 \mathrm{a}\end{array}$ & $\begin{array}{c}12.67 \\
\pm 0.88 \mathrm{a}\end{array}$ \\
\hline After parturition & $\begin{array}{c}46.33 \\
\pm 1.86 \mathrm{~d}\end{array}$ & & $\begin{array}{l}3.67 \\
1.76 \mathrm{c}\end{array}$ & $\begin{array}{c}21.00 \\
\pm 0.58 \mathrm{c}\end{array}$ & $\begin{array}{c}12.67 \\
\pm 0.88 \mathrm{ab}\end{array}$ & $\begin{array}{c}7.67 \\
\pm 0.88 \mathrm{c}\end{array}$ & $\begin{array}{c}44.67 \\
\pm 0.88 \mathrm{e}\end{array}$ & $\begin{array}{l}52.00 \\
\pm 1.155 \mathrm{~d}\end{array}$ & $\begin{array}{c}39.33 \\
\pm 0.88 \mathrm{~d}\end{array}$ & $\begin{array}{c}15.33 \\
\pm 1.20 \mathrm{a}\end{array}$ & $\begin{array}{c}10.03 \\
\pm 1.53 \mathrm{~b}\end{array}$ \\
\hline LSD at $5 \%$ & 4.36 & & .79 & 5.79 & 3.12 & 3.19 & 5.27 & 4.60 & 3.93 & 3.29 & 4.23 \\
\hline \multicolumn{12}{|c|}{$\begin{array}{l}\mathrm{CHO}=\text { Total Cholesterol, } \mathrm{TG}=\text { Triglycerides, } \mathrm{LDL}=\text { Low Density Lipoprotein, } \mathrm{HDL}=\text { High Density Lipoprotein, } \mathrm{VLDL}=\text { Very Low Density Lipoprotein; Control group: Fed on } \\
\text { basal diet. Group 1: Fed on yogurt culture. Group 2: Fed on yogurt culture + B. breve. Group 3: Fed on yogurt culture + Lb. paracasei, B. breve. }\end{array}$} \\
\hline Treatments & & egative co & atrol & & Grou & up 1 & & Group & & Gro & up 3 \\
\hline Before pregnancy & 6186 & $36.67 \pm 26$ & $1.589 \mathrm{c}$ & & $7466.67 \pm$ & $290.585 \mathrm{c}$ & & $7150.00 \pm 8$ & $6.600 \mathrm{~d}$ & $9300.00=$ & $115.467 \mathrm{c}$ \\
\hline 1st week pregnancy & & $56.67 \pm 105$ & $880 \mathrm{c}$ & & $7583.33 \pm$ & $=109.288 \mathrm{~d}$ & & $9483.33 \pm 10$ & $9.288 \mathrm{a}$ & 9850.00 & $\pm 86.600 \mathrm{~b}$ \\
\hline 2nd week pregnancy & & $34.3 \pm 44$ & 095a & & $9600.00 \pm$ & $=305.496 \mathrm{~b}$ & & $8833.33 \pm 21$ & $8.575 b$ & 10150.00 & $\pm 76.374 \mathrm{~b}$ \\
\hline 3rd week pregnancy & 8200 & $0.00 \pm 43$ & $.878 b$ & & $10443.33 \pm$ & $\pm 127.319 \mathrm{a}$ & & $8366.67 \pm 31$ & $7.971 \mathrm{bc}$ & 11300.00 & $\pm 230.934 \mathrm{a}$ \\
\hline After parturition & 5401 & $1.67 \pm 29$ & $.162 \mathrm{c}$ & & $9600.00 \pm$ & $=305.496 \mathrm{~b}$ & & $7950.00 \pm 7$ & $6.374 \mathrm{c}$ & 10033.33 & $\pm 120.182 b$ \\
\hline LSD at $5 \%$ & & 848.6 & & & & 70.906 & & 588. & 105 & & 32.072 \\
\hline
\end{tabular}

Control group: Fed on basal diet. Group 1: Fed on yogurt culture. Group 2: Fed on yogurt culture + B. breve. Group 3: Fed on yogurt culture + Lb. paracasei, B. breve.

The increase continued through gestation particularly in group 1 followed by group 3 . The counts after parturition in the probiotic groups were significantly higher than before pregnancy which is contrary to the control. The counts were 7446, 7150 and 9300 before pregnancy and were 10443, 8336 and 11300 cells on third week of gestation and reached 9600, 7950 and 10033 after parturition in groups 1, 2 and 3 respectively. Literature pointed out that in pregnancy white blood cells (WBC) in creased significantly in humans (from $6 \times 10$ into $12 \times 106 / \mathrm{L}$ ) but the increase was not that high in the rats [8] (from 7 $\times 106$ into $9 \times 106 / \mathrm{L})$. However, results pointed out that probiotics feeding significantly increased leucocytes on pregnancy particularly in group one, in which leucocytes increased from $7.5 \times 103$ into $10.5 \times 103$.

Table 7 illustrates lymphocyte percent of white blood cells in the experimental rats as changed by pregnancy. Before pregnancy, lymphocyte percent of all the groups 
Table 7. The effect of feeding probiotics on rats' lymphocytes and T-cells percent of white blood cells during pregnancy and after parturition.

\begin{tabular}{|c|c|c|c|c|c|c|c|c|}
\hline \multirow{2}{*}{ Treatments } & \multicolumn{4}{|c|}{ Negative control } & \multicolumn{4}{|c|}{ Group 1} \\
\hline & Lymp. & $\mathrm{CD}_{3}$ & $\mathrm{CD}_{4}$ & $\mathrm{CD}_{8}$ & Lymp. & $\mathrm{CD}_{3}$ & $\mathrm{CD}_{4}$ & $\mathrm{CD}_{8}$ \\
\hline Before pregnancy & $\begin{array}{c}40.33 \\
\pm 0.88 \mathrm{a}\end{array}$ & $\begin{array}{c}0.15 \\
\pm 0.02 \mathrm{a}\end{array}$ & $\begin{array}{c}50.33 \\
\pm 1.20 \mathrm{a}\end{array}$ & $\begin{array}{c}45.67 \\
\pm 2.33 \mathrm{a}\end{array}$ & $\begin{array}{c}55.33 \\
\pm 2.91 \mathrm{ab}\end{array}$ & $\begin{array}{c}1.37 \\
\pm 0.09 \mathrm{a}\end{array}$ & $\begin{array}{r}63.67 \\
\pm 1.33 \mathrm{bc}\end{array}$ & $\begin{array}{r}32.00 \\
\pm 1.15 \mathrm{~b}\end{array}$ \\
\hline 1st week pregnancy & $\begin{array}{r}32.33 \\
\pm 1.45 \mathrm{~b}\end{array}$ & $\begin{aligned} & 0.09 \\
\pm & 0.01 \mathrm{~b}\end{aligned}$ & $\begin{array}{r}42.00 \\
\pm 1.15 \mathrm{~b}\end{array}$ & $\begin{array}{r}32.00 \\
\pm 1.53 \mathrm{c}\end{array}$ & $\begin{array}{c}53.67 \\
\pm 1.76 \mathrm{~b}\end{array}$ & $\begin{array}{c}0.99 \\
\pm 0.05 \mathrm{c}\end{array}$ & $\begin{array}{c}61.00 \\
\pm 0.58 \mathrm{c}\end{array}$ & $\begin{array}{c}31.67 \\
\pm 2.19 \mathrm{~b}\end{array}$ \\
\hline $\begin{array}{l}\text { 2nd week preg- } \\
\text { nancy }\end{array}$ & $\begin{array}{c}42.00 \\
\pm 1.53 \mathrm{a}\end{array}$ & $\begin{aligned} & 0.10 \\
\pm & 0.01 \mathrm{~b}\end{aligned}$ & $\begin{array}{c}40.33 \\
\pm 0.88 \mathrm{~b}\end{array}$ & $\begin{array}{c}37.33 \\
\pm 1.20 \mathrm{bc}\end{array}$ & $\begin{array}{c}50.33 \\
\pm 0.88 \mathrm{~b}\end{array}$ & $\begin{aligned} & 1.16 \\
\pm & 0.02 b\end{aligned}$ & $\begin{array}{c}65.67 \\
\pm 1.20 \mathrm{~b}\end{array}$ & $\begin{array}{c}33.67 \\
\pm 1.86 \mathrm{ab}\end{array}$ \\
\hline $\begin{array}{l}\text { 3rd week preg- } \\
\text { nancy }\end{array}$ & $\begin{array}{l}42.00 \\
\pm 1.15 \mathrm{a}\end{array}$ & $\begin{aligned} & 0.15 \\
\pm & 0.003 \mathrm{a}\end{aligned}$ & $\begin{array}{c}37.67 \\
\pm 1.86 \mathrm{~b}\end{array}$ & $\begin{aligned} & 35.00 \\
\pm & 2.52 \mathrm{bc}\end{aligned}$ & $\begin{array}{r}53.00 \\
\pm 1.15 b\end{array}$ & $\begin{aligned} & 1.12 \\
\pm & 0.01 \mathrm{bc}\end{aligned}$ & $\begin{array}{c}70.33 \\
\pm 0.88 \mathrm{a}\end{array}$ & $\begin{array}{c}34.33 \\
\pm 0.88 \mathrm{ab}\end{array}$ \\
\hline After parturition & $\begin{array}{l}37.67 \\
\pm 1.45 \mathrm{a}\end{array}$ & $\begin{array}{c}0.16 \\
\pm 0.01 \mathrm{a}\end{array}$ & $\begin{array}{c}38.00 \\
\pm 1.15 \mathrm{~b}\end{array}$ & $\begin{array}{c}40.00 \\
\pm 0.58 \mathrm{~b}\end{array}$ & $\begin{array}{c}60.00 \\
\pm 1.15 \mathrm{a}\end{array}$ & $\begin{aligned} & 1.16 \\
\pm & 0.01 \mathrm{~b}\end{aligned}$ & $\begin{array}{r}72.00 \\
\pm 1.53 \mathrm{a}\end{array}$ & $\begin{array}{c}37.33 \\
\pm 0.88 \mathrm{a}\end{array}$ \\
\hline LSD at $5 \%$ & 4.15 & 0.035 & 4.07 & 5.62 & 5.46 & 0.15 & 3.64 & 4.70 \\
\hline
\end{tabular}

\begin{tabular}{|c|c|c|c|c|c|c|c|c|}
\hline \multirow{2}{*}{ Treatments } & \multicolumn{4}{|c|}{ Group 2} & \multicolumn{4}{|c|}{ Group 3} \\
\hline & Lymp. & $\mathrm{CD}_{3}$ & Lymp. & $\mathrm{CD}_{3}$ & Lymp. & $\mathrm{CD}_{3}$ & Lymp. & $\mathrm{CD}_{3}$ \\
\hline Before pregnancy & $\begin{array}{r}62.67 \\
\pm 1.20 \mathrm{a}\end{array}$ & $\begin{aligned} & 1.05 \\
\pm & 0.01 \mathrm{~cd}\end{aligned}$ & $\begin{array}{c}62.67 \\
\pm 1.20 \mathrm{a}\end{array}$ & $\begin{aligned} & 1.05 \\
\pm & 0.01 \mathrm{~cd}\end{aligned}$ & $\begin{array}{c}62.67 \\
\pm 1.20 \mathrm{a}\end{array}$ & $\begin{aligned} & 1.05 \\
\pm & 0.01 \mathrm{~cd}\end{aligned}$ & $\begin{array}{r}62.67 \\
\pm 1.20 \mathrm{a}\end{array}$ & $\begin{aligned} & 1.05 \\
\pm & 0.01 \mathrm{~cd}\end{aligned}$ \\
\hline 1st week pregnancy & $\begin{array}{c}54.67 \\
\pm 0.88 \mathrm{~b}\end{array}$ & 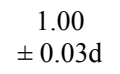 & $\begin{array}{c}54.67 \\
\pm 0.88 \mathrm{~b}\end{array}$ & $\begin{array}{l}1.00 \\
\pm 0.03 \mathrm{~d}\end{array}$ & $\begin{array}{c}54.67 \\
\pm 0.88 \mathrm{~b}\end{array}$ & $\begin{aligned} & 1.00 \\
\pm & 0.03 \mathrm{~d}\end{aligned}$ & $\begin{array}{r}54.67 \\
\pm 0.88 \mathrm{~b}\end{array}$ & $\begin{array}{l}1.00 \\
\pm 0.03 \mathrm{~d}\end{array}$ \\
\hline $\begin{array}{l}\text { 2nd week preg- } \\
\text { nancy }\end{array}$ & $\begin{array}{c}60.33 \\
\pm 0.88 \mathrm{a}\end{array}$ & $\begin{aligned} & 1.08 \\
\pm & 0.01 \mathrm{bc}\end{aligned}$ & $\begin{array}{c}60.33 \\
\pm 0.88 \mathrm{a}\end{array}$ & $\begin{aligned} & 1.08 \\
\pm & 0.01 \mathrm{bc}\end{aligned}$ & $\begin{array}{c}60.33 \\
\pm 0.88 \mathrm{a}\end{array}$ & $\begin{aligned} & 1.08 \\
\pm & 0.01 \mathrm{bc}\end{aligned}$ & $\begin{array}{c}60.33 \\
\pm 0.88 \mathrm{a}\end{array}$ & $\begin{aligned} & 1.08 \\
\pm & 0.01 \mathrm{bc}\end{aligned}$ \\
\hline $\begin{array}{l}\text { 3rd week preg- } \\
\text { nancy }\end{array}$ & $\begin{array}{r}63.33 \\
\pm 1.45 \mathrm{a}\end{array}$ & $\begin{aligned} & 1.12 \\
\pm & 0.02 \mathrm{ab}\end{aligned}$ & $\begin{array}{c}63.33 \\
\pm 1.45 \mathrm{a}\end{array}$ & $\begin{aligned} & 1.12 \\
\pm & 0.02 \mathrm{ab}\end{aligned}$ & $\begin{array}{r}63.33 \\
\pm 1.45 \mathrm{a}\end{array}$ & $\begin{aligned} & 1.12 \\
\pm & 0.02 \mathrm{ab}\end{aligned}$ & $\begin{array}{r}63.33 \\
\pm 1.45 \mathrm{a}\end{array}$ & $\begin{aligned} & 1.12 \\
\pm & 0.02 \mathrm{ab}\end{aligned}$ \\
\hline After parturition & $\begin{array}{c}64.33 \\
\pm 1.45 \mathrm{a}\end{array}$ & $\begin{aligned} & 1.14 \\
\pm & 0.01 \mathrm{a}\end{aligned}$ & $\begin{array}{c}64.33 \\
\pm 1.45 \mathrm{a}\end{array}$ & $\begin{aligned} & 1.14 \\
\pm & 0.01 \mathrm{a}\end{aligned}$ & $\begin{array}{c}64.33 \\
\pm 1.45 \mathrm{a}\end{array}$ & $\begin{aligned} & 1.14 \\
\pm & 0.01 \mathrm{a}\end{aligned}$ & $\begin{array}{c}64.33 \\
\pm 1.45 \mathrm{a}\end{array}$ & $\begin{aligned} & 1.14 \\
\pm & 0.01 \mathrm{a}\end{aligned}$ \\
\hline LSD at $5 \%$ & 3.79 & 0.05 & 3.79 & 0.05 & 3.79 & 0.05 & 3.79 & 0.05 \\
\hline
\end{tabular}

Lymp. = Lymphocyte; Control group: Fed on basal diet. Group 1: Fed on yogurt culture. Group 2: Fed on yogurt culture + B. breve. Group 3: Fed on yogurt culture + Lb. paracasei, B. breve.

ranged from $40 \%$ to $62 \%$, and this was normal for rats which are usually a lot higher than the ratio in humans (about 25\%) [8]. Lymphocyte percent in rats decreased significantly from $40.3 \%, 62.6 \%$ and $50.6 \%$ into $32.3 \%$, $54.6 \%$ and $41.6 \%$ in the control, group two and group three and insignificantly in group one on the first week of pregnancy then started to increase slightly. The ratio on the third week was insignificantly higher than ratio before pregnancy. After parturition, the percentages in the probiotic groups were insignificantly higher than before pregnancy $(60 \%, 64 \%$ and $55 \%$ in groups 1,2 and three, respectively) contrary to the control percentage which was lower than before pregnancy. The reduction in lymphocyte $\%$ on pregnancy was slight compared to literature which showed a significant reduction from $73 \%$ into $45 \%$ in rats [4].

Table 8 illustrates absolute counts of lymphocytes and phagocytes as calculated from lymphocyte percentage of leucocytes. The control on first week of pregnancy showed a significant $(\mathrm{p}<0.05)$ reduction in lymphocytes with no change in phagocytes and on the second week of gestation, both counts significantly increased and on the third week, the counts were significantly higher than before pregnancy. Lymphocyte and phagocyte counts were 2526 and 3750 before pregnancy and 3440 and 4760 cells $/ \mathrm{ml}$ on third week of pregnancy, respectively. However, after parturition the counts were significantly lower than before pregnancy (2041 lymphocytes and 3360 phagocytes). Group one showed lymphocyte insignificant reduction on first week of pregnancy with a slight increase in phagocyte cells, then both counts started to increase to be on the third week significantly higher than before pregnancy. The same significant difference existed after parturition. Lymphocyte and phagocyte counts were 4148 and 3318 before pregnancy and increased to 5532 and 4911 on third week of gestation and 5766 and 3833 cells/ml after parturition. Group two showed a significant increase in both types of cells, particularly the phagocytes on first week of gestation and, the increase continued even after parturition.

Both cells were higher than before pregnancy and the difference was significant in Lymphocyte. Lymphocyte 
Table 8. The Effect of feeding probiotics on lymphocytes and phagocytes absolute count (cell/ml) of rats during pregnancy and after parturition.

\begin{tabular}{|c|c|c|c|c|c|c|c|c|}
\hline \multirow{2}{*}{ Treatments } & \multicolumn{2}{|c|}{ Negative control } & \multicolumn{2}{|c|}{ Group 1} & \multicolumn{2}{|c|}{ Group 2} & \multicolumn{2}{|c|}{ Group 3} \\
\hline & Lymphocyte & Phagocyte & Lymphocyte & Phagocyte & Lymphocyte & Phagocyte & Lymphocyte & Phagocyte \\
\hline 1st week pregnancy & $\begin{array}{c}1797.67 \\
\pm 98.95 \mathrm{~d}\end{array}$ & $\begin{array}{l}3759.00 \\
\pm 89.61 \mathrm{c}\end{array}$ & $\begin{array}{c}4069.95 \\
\pm 110.31 \mathrm{c}\end{array}$ & $\begin{array}{c}3513.43 \\
\pm 139.20 \mathrm{c}\end{array}$ & $\begin{array}{c}5184.67 \\
\pm 116.64 \mathrm{a}\end{array}$ & $\begin{array}{l}4298.33 \\
\pm 81.59 \mathrm{a}\end{array}$ & $\begin{array}{c}4103.67 \\
\pm 115.35 \mathrm{c}\end{array}$ & $\begin{array}{c}5746.33 \\
\pm 140.20 \mathrm{a}\end{array}$ \\
\hline 2nd week pregnancy & $\begin{array}{c}2996.33 \\
\pm 161.65 \mathrm{a}\end{array}$ & $\begin{array}{c}5848.00 \\
\pm 146.53 \mathrm{a}\end{array}$ & $\begin{array}{l}4826.67 \\
\pm 73.33 b\end{array}$ & $\begin{array}{c}4106.67 \\
\pm 530.44 \mathrm{ab}\end{array}$ & $\begin{array}{c}5332.67 \\
\pm 200.87 \mathrm{a}\end{array}$ & $\begin{array}{l}3500.67 \\
\pm 49.83 b\end{array}$ & $\begin{array}{c}4805.33 \\
\pm 145.21 \mathrm{~b}\end{array}$ & $\begin{array}{c}5344.67 \\
\pm 105.45 \mathrm{a}\end{array}$ \\
\hline 3rd week pregnancy & $\begin{array}{c}3440.00 \\
\pm 170.88 \mathrm{~b}\end{array}$ & $\begin{array}{c}4760.00 \\
\pm 298.66 \mathrm{~b}\end{array}$ & $\begin{array}{l}5532.00 \\
\pm 57.00 \mathrm{a}\end{array}$ & $\begin{array}{c}4911.00 \\
\pm 178.80 \mathrm{a}\end{array}$ & $\begin{array}{c}5307.67 \\
\pm 322.64 \mathrm{a}\end{array}$ & $\begin{array}{l}3059.00 \\
\pm 35.51 \mathrm{c}\end{array}$ & $\begin{array}{c}5911.00 \\
\pm 160.82 \mathrm{a}\end{array}$ & $\begin{array}{c}5389.00 \\
\pm 234.37 \mathrm{a}\end{array}$ \\
\hline After parturition & $\begin{array}{c}2041.33 \\
\pm 184.76 \mathrm{~d}\end{array}$ & $\begin{array}{c}3360.33 \\
\pm 127.32 \mathrm{c}\end{array}$ & $\begin{array}{c}5766.67 \\
\pm 288.51 \mathrm{a}\end{array}$ & $\begin{array}{c}3833.33 \\
\pm 43.72 \mathrm{bc}\end{array}$ & $\begin{array}{c}5116.33 \\
\pm 160.80 \mathrm{a}\end{array}$ & $\begin{array}{c}2833.33 \\
\pm 91.92 \mathrm{~cd}\end{array}$ & $\begin{array}{c}5521.00 \\
\pm 179.78 \mathrm{a}\end{array}$ & $\begin{array}{l}4512.33 \\
\pm 65.57 \mathrm{~b}\end{array}$ \\
\hline LSD at $5 \%$ & 453.84 & 606.92 & 697.34 & 825.46 & 624.31 & 227.04 & 578.64 & 480.30 \\
\hline
\end{tabular}

Control group: Fed on basal diet. Group 1: Fed on yogurt culture. Group 2: Fed on yogurt culture + B. breve. Group 3: Fed on yogurt culture + Lb. paracasei, B. breve.

and phagocyte counts increased from 4481 and 2668 before pregnancy into 5307 and 3059 on third week of pregnancy and 5116 and 2833 cells/ml after parturition, respectively. Once again, in group three there was significant lymphocytes reduction on first week of pregnancy accompanied with significant increase in phagocytes, then followed by the increase in both cells. In all probiotic groups, lymphocyte counts on third week of pregnancy and after parturition were significantly higher than before pregnancy. In group 3 for example, lymphocyte counts increased from 4717 before pregnancy into 5911 and 5521 cells $/ \mathrm{ml}$ on third week of gestation period and after parturition, respectively. On other hand, phagocytes were higher with significant difference on third week but the difference was insignificant after parturition compared to the level before pregnancy. During gestation, counts of both cells were enhanced by probiotic feeding and the increase favored lymphocyte counts than the control. The enhancement in phagocytes is in harmony with the opinion that pregnancy has an inflammatory response. Compared to the control both sides of the immune response were stimulated with probiotics feeding, the innate (phagocytes) and cell mediated (lymphocytes) and the enhancing effect of probiotics continued after parturition.

Table 7 points out the $\mathrm{CD}_{4+}$ (T-helper) percentages of lymphocytes during pregnancy and postpartum. Cells percentage decreased on first week of pregnancy in all the groups, and then the probiotics behaved differently than the control. Control group showed significant reduction on first week of pregnancy then the decrease continued with a slower rate through gestation to end with a significant lower percentage than before pregnancy. The counts started with 50.3 and decreased to 37.6 and 38 cells $/ \mathrm{ml}$ on third week of pregnancy and after parturition, respectively. The probiotic groups showed insignificant $(p<0.05)$ decrease on pregnancy followed by progressive increase through gestation and postpartum. In yogurt culture group, $\mathrm{CD}_{4+}$ percentage on the third week of pregnancy $(70.3 \%)$ and after parturition were significantly higher $(72 \%)$ than before pregnancy $(63.6 \%)$ while in groups two and three the increase was insignificant. So, compared to the control, probiotics feeding helped $\mathrm{CD}_{4+}$ to regain their level before pregnancy and on the third week of gestation $\mathrm{CD}_{4+}$ was higher than before pregnancy. The effect of pregnancy on $\mathrm{CD}_{4+}$ varied in literature from almost no change in T-helper (Th), T-cell (Tc), Natural Killer (NK) and Interleukin-4 (IL-4) in rats during pregnancy and others reported a reduction in their counts. The reduction in T-helper was reported as a result of the decrease in thymus gland (thymic involution) during pregnancy in human and mice. T-cell development is blocked at the precursor level during pregnancy, this process continued during lactation with selective loss of $\mathrm{CD}_{4+}$ and $\mathrm{CD}_{8+}$ with a gradual recovery in the postpartum period [24]. Thus, the progressive increase during gestation in $\mathrm{CD}_{4+}$ by probiotics feeding as compared to the control would mean that they enhanced thymus gland recovery.

$\mathrm{CD}_{8+}(\mathrm{Tc})$ which includes suppressor and killer or cytoxic cells behaved similar to $\mathrm{CD}_{4+}$. The control showed significant reduction on pregnancy (from 45.6 into 32\%) and the decrease continued during gestation and after parturition, to end with significantly lower percentage than before pregnancy. Probiotic groups showed a first week reduction followed by a progressive increase during gestation to end with significantly higher percentage on postpartum, particularly in group one and three. The 
percentage increased from 32,43 and 38.3 before pregnancy into $37.3,46.3$ and 54.6 after parturition. The $\mathrm{CD}_{4} / \mathrm{CD}_{8}$ ratios in all the groups were within the normal values $(1.1-2.9)$ with no significant changes.

In conclusion probiotics, compared to the control, enhanced leucocytes production particularly group three (yogurt culture plus $B$. breve plus $L b$. paracasei) followed by group two (yogurt culture plus B. breve), both groups did not even exert the dip in leucocytes on first week of pregnancy as occurred in the control. The enhancement continued postpartum in the three groups. The increase in leucocytes distributed differently over the counts of lymphocytes, phagocytes, $\mathrm{CD}_{4+}$ and $\mathrm{CD}_{8+}$. Contrarily to the control where their leucocytes increase on pregnancy favored phagocytes count, the significant increase by probiotics favored lymphocytes count. $\mathrm{CD}_{4+}$ and $\mathrm{CD}_{8+}$ counts were enhanced by probiotic feeding. The count dip, which occurred on first week, was significant in the control and insignificant in probiotic groups. This insignificant reduction was followed by progressive increase to achieve significantly higher counts on the second or third week of pregnancy than before pregnancy counts.

After parturition, probiotics feeding greatly helped the counts of lymphocytes, $\mathrm{CD}_{4+}$ and $\mathrm{CD}_{8+}$ to be higher than before pregnancy and mostly with significant differences.

These results pointed out that feeding probiotics enhanced both arms of the immunity on pregnancy. The enhancement in cell mediated response would help in overcoming the increased susceptibility for infection as a result of the down regulating of $\mathrm{CD}_{4+}$ and $\mathrm{CD}_{8+}$ cells accompanied pregnancy.

\section{Acknowledgements}

We thank Prof. Mohamed M. E. Metwally for his valuable help and support in getting this work done. This research did not receive any specific grant from any funding body. It's done as a part of the research plan of the Dairy science and Technology Department, Faculty of Agriculture, Cairo University. The authors' responsibilities were as follows: A. A. A. and A. M. M. M. designed the entire study and contributed to the execution of the experimental work. A. H. M. and H. F. A. supervised the cultures preparation, rats feeding and blood samples collection and analysis. All authors approved the final manuscript. All authors declared that they had no conflict of interest.

\section{REFERENCES}

[1] P. Luppi, "How Immune Mechanisms are Affected by Pregnancy," Vaccine, Vol. 21, No. 24, 2003, pp. 33523357. doi:10.1016/S0264-410X(03)00331-1
[2] T. E. Ivanova, M. T. S. R. Aivazova, A. Nallansk, T. Chernev and D. S. Kiurkchiev, "Changes of Activation in T-Lymphocyte Subpopulations in Peripheral Blood during Pregnancy," Akush Ginekol (Sofira), Vol. 46, 2007, pp. 3-8.

[3] S. J. Orange and Z. K. Ballas, "Natural Killer Cells in Human Health and Disease," Clinical Immunology, Vol. 118, No. 1, 2006, pp. 1-10. doi:10.1016/j.clim.2005.10.011

[4] M. M. Faas, A. Bouman, L. Angelique, N. Veenstra, S. Gerda, M. Henk, H. J. Maas and V. Paul, "Species Differences in the Effect of Pregnancy on Lymphocyte Cytokine Production between Human and Rat," Journal of Leukocyte Biology, Vol. 78, No. 4, 2005, pp. 946-953. doi:10.1189/jlb.0405186

[5] L. M. Andrew and M. David, "Policing Pregnancy: Tregs Keep the Peace," Trends in Iimmunology, Vol. 25, 2004, pp. 563-565. doi:10.1016/j.it.2004.09.001

[6] C. E. Lewis, "Adverse Effect of Pregnancy on High Density Lipoprotein (HDL) Cholesterol in Young Adult Women," American Journal of Epidemiology, Vol. 144, 1996, pp. 247-254.

[7] M. V. Tejada-Simon, H. J. Lee, Z. Ustunol and J. J. Pestka, "Ingestion of Yogurt Containing Lactobacillus Acidophilus and Bifidobacterium to Potentiate Immunoglobulin: A Responses to Cholera Toxin in Mice," Journal of Dairy Science, Vol. 82, 1999, pp. 649-660. doi:10.3168/jds.S0022-0302(99)75281-1

[8] E. J. Schiffrin, F. Rochat, H. Link-Amster, J. M. Aeschlimann and A. Donnet-Hughes, "Immunomodulation of Human Blood Cells Following the Ingestion of Lactic Acid Bacteria," Journal of Dairy Science, Vol. 78, No. 3, 1995, pp. 491-497. doi:10.3168/jds.S0022-0302(95)76659-0

[9] A. S. Akaline, G. Siddik and D. Selmin, "Influence of Yogurt and Acidophilus Yogurt on Serum Cholesterol Levels in Mice," Journal of Dairy Science, Vol. 80, No. 11, 1996, pp. 2721-2725.

[10] J. Z. Xiao, S. Kondo, N. Takahashi, K. Miyaji, K. Oshidaf, A. Hiramatsu, K. Iwatsuki, S. Kokubo and A. Hosonof, "Effects of Milk Products Fermented by Bifidobacterium Longum on Blood Lipids in Rats and Healthy Adult Male Volunteers," Journal of Dairy Science, Vol. 86, No. 7, 2003, pp. 2452-2461. doi:10.3168/jds.S0022-0302(03)73839-9

[11] E. Laffineu, N. Genetet and J. Leonil, "Immunomodulatory Activity of B-Casein Permeate Medium Fermented by Lactic Acid Bacteria," Journal of Dairy Science, Vol. 79, 1996, pp. 2112-2120. doi:10.3168/jds.S0022-0302(96)76585-2

[12] E. J. Ko, J. S. Goh, B. J. Lee, S. H. Choi and P. H. Kim, "Bifidobacterium Bifidum Exhibits a Lipopolysaccharide-Like Mitogenic Activity for Murine B Lymphocytes," Journal of Dairy Science, Vol. 82, 1999, pp. 1869 -1876. doi:10.3168/jds.S0022-0302(99)75420-2

[13] J. Leblank, I. Fliss and C. Matar, "Induction of a Humoral Immune Response Following an Escherichia Coli O157:H7 
Infection with an Immunomodulatory Peptidic Fraction Derived from Lactobacillus Helveticus-Fermented Milk," American Society for Microbiology, Vol. 11, No. 6, 2004, pp. 1171-1181.

[14] J. G. LeBlanc, C. Matar, J. C. Valdez, J. LeBlanc and G. Perdigon, "Immunomodulating Effects of Peptidic Fractions Issued from Milk Fermented with Lactobacillus Helveticus," Journal of Dairy Science, Vol. 85, 2001, pp. 2733-2742. doi:10.3168/jds.S0022-0302(02)74360-9

[15] G. Perdigon, S. Alvarez, M. Rachid, G. Aguero and N. Gobbato, "Immune System Stimulation by Probiotics," Journal of Dairy Science, Vol. 78, No. 7, 1995, pp. 15971606. doi:10.3168/jds.S0022-0302(95)76784-4

[16] J. van de Water, C. Keen and M. E. Gershwin, "The Influence of Chronic Yogurt Consumption on Immunity," Journal of Nutrition, Vol. 129, No. 7, 1999, pp. 1492S1495S.

[17] G. Perdigon, E. Vintini, S. Alvarez, M. Medina and M. Medici, "Study of the Possible Mechanisms Involved in the Mucosal Immune System Activation by Lactic Acid Bacteria," Journal of Dairy Science, Vol. 82, No. 6, 1999 , pp. 1108-1114. doi:10.3168/jds.S0022-0302(99)75333-6

[18] T. Asahara, K. Shimizu, K. Nomoto, T. Hamabata and Y. Takeda, "Probiotic Bifidobacteria Protect Mice from Lethal Infection with Shiga Toxin-Producing Escherichia
Coli O157:H7," Infection and Immunity, Vol. 72, No. 4, 2004, pp. 2240-2247. doi:10.1128/IAI.72.4.2240-2247.2004

[19] M. Ogawa, K. Shimizu, K. Nomoto, M. Takahashi, M. Watanuki, T. Tanaka, T. Hamabata, S. Yamasaki and Y. Takeda, "Protective Effect of Lactobacillus Casei Strain Shirota on Shiga Toxin-Producing Escherichia Coli O157: H7 Infection in Infant Rabbits," Infection and Immunity, Vol. 69, No. 2, 2001, pp. 1101-1108. doi:10.1128/IAI.69.2.1101-1108.2001

[20] A. Samona and R. K. Robison, "Enumeration of Bifidobacteria in Dairy Products," Journal of Dairy Technology, Vol. 44, No. 3, 1991, pp. 64-66. doi:10.1111/j.1471-0307.1991.tb00639.x

[21] S. R. A. Fisher, "Statical method for research worker's," Oliver and Void, Vol. 14, 1970, p. 140.

[22] M. Waller and B. Dunkan, "Role for Symmetric Multiply Composition Problem," American Science Associates, Vol. 65, 1969, p. 485.

[23] SPSS for Windows, V. 12.0, SPSS Inc., Copyright $(2003$, Chicago, IL, USA.

[24] A. G. Rijhsinghani, S. K. Bhatia, T. Tygrettle and T. Waldschmildt, "Effect of Pregnancy on Thymic T Cells Developed," American Journal of Reproductive Immunology, Vol. 35, 1996, pp. 523-528. 